\title{
Targeted Molecular Therapy
}

National Cancer Institute

\section{Source}

National Cancer Institute. Targeted Molecular Therapy. NCI Thesaurus. Code C76243.

Cancer therapies designed to act upon specific molecules in metabolic pathways or processes involved in carcinogenesis, tumor growth, or tumor spread. 